\title{
Genetic Variability of Traits Related to Synchronous Maturity in Greengram [Vigna radiata (L.) Wilczek]
}

\author{
Nivedita Talukdar ${ }^{1 *}$, Hironyo Kr. Borah ${ }^{2}$ and Ramendra Nath Sarma ${ }^{3}$ \\ ${ }^{1}$ Department of Plant Breeding and Genetics, Assam Agricultural University, Jorhat, Assam \\ ${ }^{2}$ Plant Breeding and Genetics, Regional Agricultural Research Station, Assam Agricultural \\ University, Shillongoni \\ ${ }^{3}$ Department of Plant Breeding and Genetics, Assam Agricultural University, \\ Jorhat, Assam, India \\ *Corresponding author
}

\section{Keywords}

Greengram, variability, Synchrony, $\mathrm{D}^{2}$ analysis, Genetic divergence

Article Info

Accepted: 15 December 2019 Available Online: 20 January 2020

\section{A B S T R A C T}

The present study was conducted to evaluate 38 greengram genotypes to determinethe nature and extent of variation in the phenological traits related to synchronous maturity. A field trial was laid under a Randomized Block Design (RBD) with three replications; observations were recorded on 17 morphological characters. Analysis of variance revealed significant variation among genotypes for all the characters except for 100 seed weight. Different genotypes were found superior for different characters. Phenotypic coefficient of variation (PCV) was greater than that of Genotypic coefficient of variation (GCV) for all characters studied thereby indicating the influence of environmental effect on the characters. The GCV and PCV estimates were high for number of pods per plant followed by degree of indetermination of plant height from first pod maturity to $90 \%$ pod maturity and number of branches per plant respectively. High heritability coupled with high genetic advanced as percent of mean was observed for 13 traits including yield per plant. Based on $\mathrm{D}^{2}$ values, the genotypes were grouped into eight clusters. Maximum inter cluster distance was observed between clusters III and cluster VI followed by cluster Vand cluster VIII while lowest distance was noticed between cluster I and cluster VII. Days to $90 \%$ pod maturity followed by days to first flowering and days to first pod maturity had highest contribution towards genetic divergence.

\section{Introduction}

Greengram or Mungbean (Vigna radiata L. Wilczek) is a self pollinated diploid $(2 \mathrm{n}=22)$ pulse crop native to the Indian subcontinent. It contains about 25 per cent protein, which is almost three times that of cereals. India ranks first both in acreage and production of greengram in the world. During 2017-18, Greengram was sown over an area of 4.26 Mha in (kharif + rabi) and recorded a production of $2.01 \mathrm{Mt}$ at and yield level of $472 \mathrm{~kg} / \mathrm{ha}$ (Anon., 2018). Rajasthan, MP, Maharashtra, Karnataka, Bihar, AP, Odisha, 
Tamil Nadu, Gujarat and Telangana have been the major states. But productivity of the crop is very low to meet the requirement of India's ever increasing population.

Greengram shows asynchronous pattern of maturity, which is not desired by farmers as it requires several pickings for harvesting of the crop and make it a labour intensive, costly practice. Moreover, asynchrony in flowering is also attributed to lower productivity due to high harvest index. Therefore, morphophysiological responses in flower production and flowering pattern that ultimately leads to more mature pods and final yield needs to be properly assessed. A synchronous variety being short duration is suitable to fit in any cropping system without direct competition to the main crops. This suggests that understanding of flowering pattern is useful in the selection of high yielding genotypes. There is however little information on its flowering behaviour.

Pod maturity in mungbean is not synchronous (Yeates et al., 2000) and this is reported to be one of the causes for low yield and low harvest index in mungbean (Bushby and Lawn, 1992; Egli and Bruening, 2006). Asynchrony is linked to indeterminate growth habit resulting in simultaneous transformation of assimilates into both vegetative and reproductive parts which can explain low harvest index in such crops. Again, it is observed that earlier formed pods are heavier than later formed pods indicating inadequate supply of assimilates to later formed pods (Fakir, 1997; Kuroda et al., 1998; Begum et al., 2007). Inverse effects of high leafiness and asynchronous flowering on seed yield were further reported by Bisht et al., (1998 \& 2005). Although the new mungbean varieties are not true determinate, technically they are also not indeterminate and the pre and postflowering plant height variation in Mungbean has been described as the degree of indetermination of plant height (Khattak et al., 2001). Information on the degree of indetermination of growth duration could help in adopting suitable and efficient strategies to develop mungbean genotypes with synchronous growth habit coupled with high yield since time of pod maturation is an important factor in synchronous pod maturity (Sharma-Natu and Ghildiyal, 2005). Moreover, genotype that produces maximum opened flower within 10-15 days and ceases flowering after 15-20 days is desirable. Breeding effort towards the development of synchronous maturity in greengram is limited. For example, out of 40 greengram varieties released of CVRC in 2017-18, only one variety (VBN8) was found to be determinate type (Anon., 2019), indicating limitations in breeding effort. However, these results indicate potentiality of developing such a variety using existing genetic resources and modern tools of crop improvement.

\section{Materials and Methods}

The experimental material consisted of 38 green gram genotypes (Vigna radiata (L.) Wilczek) obtained from institutes from all over India and was collected from RARS, Shillongoni. The genotypes used were ML 818, KM 2241, MH 2-15, OUM 11-5, HUM 1, COGG 912, Pant M-5, Pusa 0672, Pant M4, IPM 02-3, BM 4, AKM 8802, IPM 2-14, IPM 312-19, IPM 312-20, IPM 14-7, GGG 1, MH 1142, RMG 1087, RMG 1092, COGG 13-19, PM 14-3, PM 14-11, AKM 12-28, AKM 12-24, TMB 126, SKNM 1502, SKNM 1504, LGG 607, JAUM 0936, NMK 15-08, KM 2355, ML 2479, SML 1808, COGG 1339, RMG 1097, SGC 16 and SGC 20. Each genotype was sown in rows of 4 metres length, adopting a spacing of $30 \times 10 \mathrm{~cm}$ in a RBD with three replications. Other recommended packages of practices were followed to raise successful crop of greengram. Five randomly taken plants were 
considered to record data fordays to first flowering, days to $50 \%$ flowering, days to first pod maturity, days to $90 \%$ pod maturity, degree of indetermination of pod maturity from first flower to $90 \%$ pod maturity $\left(\mathrm{DDd}_{1}\right)$, degree of indetermination of pod maturity from first pod maturity to $90 \%$ pod maturity $\left(\mathrm{DDd}_{2}\right)$, plant height at first flower initiation $(\mathrm{cm})$, plant height at first pod maturity $(\mathrm{cm})$, plant height at $90 \%$ pod maturity $(\mathrm{cm})$, degree of indetermination of plant height from first flower to first pod maturity $\left(\mathrm{DDh}_{1}\right)$, degree of indetermination of plant height from first flower to $90 \%$ pod maturity $\left(\mathrm{DDh}_{2}\right)$, degree of indetermination of plant height from first pod maturity to $90 \%$ pod maturity $\left(\mathrm{DDh}_{3}\right)$, number of pods per plant, number of branches per plant, number of seeds per pod, 100 seed weight $(\mathrm{g})$ and seed yield per plant (g). Degree of indeterminations of pod maturity and plant height were calculated according to the formulae outlined by Khattak et al., (2004). The mean values of five plants were taken for the statistical analysis. Statistical methods suggested by Burton and Devane (1953) for variability, Robinson et al., (1949) for heritability, Johnson et al., (1955) for genetic advance as per cent of mean were adopted to find out the respective estimates. Mahalanobis $\mathrm{D}^{2}$ (Rao, 1952) analysis was performed in Biotool package of R software (da Silva et al., 2017).

\section{Results and Discussion}

Analysis of variance (Table 1) revealed that there were significant variations among the genotypes for all the characters except 100 seed weight. So, this indicated sufficient variations among the genotypes under study for the characters indicating scope for selection. Per se performance of different genotypes under study (Table 2) revealed that different genotypes possessed different desirable yield attributes and synchrony related phenological traits. For example, COGG 13-39 was the earliest in days to first flowering (44.00 days), but $\mathrm{MH} 2-15$ was earliest for days to $50 \%$ flowering (50.67 days). Similarly, AKM 8802 was the earliest for days to first pod maturity (54.00 days) and AKM 12-28 was earliest for days to $90 \%$ pod maturity (64.67 days). Pant M-5 was the shortest in plant stature at first flower initiation $(13.50 \mathrm{~cm})$ and at first pod maturity $(20.40 \mathrm{~cm})$, but TMB 126 was shortest at $90 \%$ pod maturity $(30.33 \mathrm{~cm})$. On the other hand, IPM 312-20 had the highest pods per plant (29.87) and branches per plant (7.67) along with IPM 312-19 (7.67). IPM 312-20, COGG 13-39 along with KM 2355 had highest seeds per pods (13.00). KM 2355 further had the highest 100 seed weight $(5.40 \mathrm{~g})$ and seed yield per plant (10.22g). When different synchrony related attributes were compared, Pant M-4 was the most determinate in terms degree of indetermination of pod maturity from first flower and first pod maturity to $90 \%$ pod maturity $(30.47$; 10.47$)$ but $\mathrm{RMG}$ 1092 was the most determinate in terms of degree of indetermination of plant height from first flower to first pod (8.38) and 90\% pod maturity (23.96) while RMG 1087 was determinate in terms of degree of indetermination of plant height from first pod maturity to $90 \%$ pod maturity (8.28). It was observed that indeterminate behaviour is not directly related to days to first flowering and days to first pod maturity. No varieties possessed all the desirable attributes for synchrony and high yield. The identified genotypes with desirable attributes may carefully be used as parents for hybridization programme to obtain desirable segregant combining better yield with synchronous flowering.

\section{Genetic components}

Phenotypic coefficient of variation (PCV) was higher than Genotypic coefficient of variation 
(GCV) for all the traits under study (Table 3) which indicated that the variation was not due only to genotypes but also due to the influence of environment. These results are in accordance with Mehandi et al., (2013), Raturi et al., (2015), Hemavathy et al., (2015) and Anand et al., (2015) Venkateswarlu (2001), Dikshit et al., (2002), Reddy et al., (2003) in greengram. In the present study, high GCV, PCV was observed for degree of indetermination of plant height from first flower to first pod maturity $\left(\mathrm{DDh}_{1}\right)$, degree of indetermination of plant height from first pod maturity to $90 \%$ pod maturity $\left(\mathrm{DDh}_{3}\right)$, number of pods per plant and number of branches per plant indicating the predominance of additive gene action. It is expected that the characters exhibiting high GCV would exhibit response to selection in a positive direction.

A high heritability $\left(\mathrm{H}^{2}\right)$ in broad sense(bs) was recorded for all the traits except for degree of indetermination of plant height from first flower to first pod maturity which showed moderate heritability. The highest estimate was observed for plant height at $90 \%$ pod maturity. The estimates of heritability are environment specific (Shimelis and Rhandzu, 2010). Thus, reliability of selection does not depend upon heritability alone as it is likely to be misleading. Hence, selection of traits based on heritability and genetic advance as percent of mean (GAM) is of great importance Mehandi et al., (2013), Ginwal et al., (2004).High heritability estimates coupled with high or moderate genetic advance was observed for 13 traits including yield per plant. Similar results were reported by (Muthuswamy et al., 2019) for plant height, number of branches per plant, number of pod per plant, 100 seed weight and seed yield per plant. (Baisakh et al., 2016) also reported plant height and pods per plant had high heritability with high genetic advance. Pavan et al., (2019) also reported similar results for pods per plant, seed yield per plant, plant height and number of branches per plant. (Idress et al., 2006) also reported similar results for primary branches, pods per plant, 100 seed weight and seed yield. Similar results for plant height, number of pods per plant and seed yield per plant were reported by (Reddy et al., 2011). Garg et al., (2017) also observed similar results for plant height, number of branches per plant, seed per pod, 100 -seed weight, number of pods per plant and seed yield. High heritability coupled with high or moderate genetic advance indicated involvement of additive gene action in the genetic control of these traits suggesting the scope for improvement through simple selection in the present breeding material. High heritability and low genetic advance for characters like days to $50 \%$ flowering, days to first pod maturity and days to $90 \%$ pod maturity indicated the predominance of nonadditive gene action suggesting that these traits could be improved by pedigree method and population approach by breeding. The present findings were not in conformity with (Payasi, 2015) who reported high heritability with low genetic advance for number of pods per cluster, number of seeds per pod, pod length and protein content.

Based on GCV, PCV, $\mathrm{H}^{2}(\mathrm{bs})$ and GAM, degree of indetermination of plant height from first pod maturity to $90 \%$ pod maturity, number of pods per plant and number of branches per plant were identified as prominent characters among the genotypes under study to consider in a breeding programme.

\section{Genetic divergence analysis}

Mahalonobis $\mathrm{D}^{2}$ statistics was used to study genetic divergence using 17 quantitative traits since it is hypothesized that divergent parents offer substantial variability in the segregating generations. Significance of Wilk's 'V' 
statistics revealed that 38 genotypes differed significantly when all the characters were considered simultaneously indicating considerable diversity among the materials. Hence further analysis was done. Tocher's method of classification, (Table 4) based on $\mathrm{D}^{2}$ estimates grouped the genotypes into eight clusters with a single largest cluster of 23 genotypes of cluster I. It was observed that genotypes originated in same place weren't clustered together indicating lack of association between geographical locations and clustering pattern. The clustering pattern was random and independent with variable number of genotypes. Lack of association between geographical origin and clustering pattern has been reported in greengram and other crops. The results are in agreement with (Singh et al., 2013; Singh et al., 2014). In the present study, clustering pattern might be attributed to their morphological differences and breeding history. Genotypes grouped into the same cluster showed little divergence from one another as the aggregate of characters measured.

Statistical distance as shown with $\mathrm{D}^{2}$ values represents the index of genetic diversity among the clusters. Maximum intra-cluster distance was observed in cluster IV with two genotypes AKM 8802 and KM 2241, indicating sufficient diversity between two genotypes within this cluster (Table 5). Minimum inter cluster distance between Cluster I and Cluster VII indicating close relationship among the genotypes and similarity for the characters. Maximum intercluster distance observed between Cluster III and Cluster VI indicating diverse nature of the genotypes.

In the present study, the existences of higher inter cluster distance than intra cluster distance indicated existence of sufficient genetic variability among the genotypes. (Garg et al., 2017) also reported more intercluster distance than intra-cluster distance in their study. Based on inter-cluster distance, careful selection of parents from different clusters may be used in a breeding programme to generate more variability. Careful selection of divergent genotype from these two clusters would produce a broad spectrum of variability in the subsequent generations enabling further selection and improvement.

Cluster mean performance for different characters showed considerable differences between all the clusters for all the characters (Table 6). Cluster II was characterized by earliest days to first flowering and days to first pod maturity. Cluster III was characterized by earliest days to $50 \%$ flowering, earliest days to $90 \%$ pods maturity, highest number of pods per plant, 100 seed weight and seed yield per plant. Cluster IV was characterized by genotypes having shortest height at $90 \%$ pod maturity. Cluster VI was characterized by genotypes exhibiting lowest degree of indetermination of plant height from first flower and first pod maturity to $90 \%$ pod maturity. Cluster VII was characterized by genotypes exhibiting lowest degree of indetermination of pod maturity from first flower and first pod maturity to $90 \%$ pod maturity and lowest degree of indetermination of plant height from first flower to first pod maturity. Also it had genotypes with the highest number of branches per plant and seeds per pods. Cluster VIII was characterized by genotypes having shortest height at first flower initiation and first pod maturity. The results suggested that intercrossing of carefully chosen genotypes from different cluster showing good mean performance might help in obtaining high yield combining synchronous maturity. According to Mishra et al., (2018), the inclusion of more diverse parents inhybridization is believed to increase the chances of obtaining better heterosis and give broad spectrum of variability insegregating generation. 
Table.1 Analysis of variance for yield and its components in greengram

\begin{tabular}{|c|c|c|c|c|c|c|c|c|c|c|c|c|c|c|c|c|c|c|}
\hline \multirow[b]{2}{*}{$\begin{array}{l}\text { Source of } \\
\text { Variations }\end{array}$} & \multirow[b]{2}{*}{ Df } & \multicolumn{17}{|c|}{ Mean Squares } \\
\hline & & $\begin{array}{l}\text { Days to 1st } \\
\text { flowering } \\
\left(D_{1}\right)\end{array}$ & $\begin{array}{l}\text { Days to } \\
\mathbf{5 0 \%} \\
\text { flowering }\end{array}$ & $\begin{array}{l}\text { Days to } \\
\text { 1st pod } \\
\text { maturity } \\
\left(D_{2}\right)\end{array}$ & DDd $_{1}$ & $\begin{array}{l}\text { Days to } \\
90 \% \text { pod } \\
\text { maturity } \\
\left(D_{3}\right)\end{array}$ & DDd $_{2}$ & $\begin{array}{l}\text { Plant } \\
\text { height at } \\
1^{\text {st }} \text { flower } \\
\text { initiation } \\
\left(\mathrm{H}_{1}\right)\end{array}$ & $\begin{array}{l}\text { Plant } \\
\text { height at } \\
\mathbf{1}^{\text {st pod }} \\
\text { maturity } \\
\left(\mathrm{H}_{2}\right)\end{array}$ & $\mathbf{D D h}_{1}$ & $\begin{array}{l}\text { Plant } \\
\text { height at } \\
90 \% \\
\text { pods } \\
\text { maturity } \\
\left(\mathrm{H}_{3}\right)\end{array}$ & $\mathbf{D D h}_{2}$ & $\mathrm{DDh}_{3}$ & $\begin{array}{l}\text { Number } \\
\text { of pods } \\
\text { per plant }\end{array}$ & $\begin{array}{l}\text { Number } \\
\text { of } \\
\text { branches } \\
\text { per plant }\end{array}$ & $\begin{array}{l}\text { Seeds } \\
\text { per } \\
\text { pods }\end{array}$ & $\begin{array}{l}100 \\
\text { seed } \\
\text { weight } \\
\text { (g) }\end{array}$ & $\begin{array}{l}\text { seed } \\
\text { yield per } \\
\text { plant }(\mathrm{g})\end{array}$ \\
\hline Replication & 2 & 0.01 & 0.55 & 0.95 & 0.06 & 0.24 & 1.41 & 1.52 & 1.18 & $\begin{array}{c}9.61 \\
* *\end{array}$ & $\begin{array}{c}4.01 \\
*\end{array}$ & 0.08 & 4.55 & $\begin{array}{c}27.29 \\
* *\end{array}$ & 1.31 & 0.17 & 0.01 & 0.04 \\
\hline Genotype & 37 & $\begin{array}{c}18.89 \\
* *\end{array}$ & $\begin{array}{c}20.56 \\
* *\end{array}$ & $\begin{array}{c}18.80 \\
* *\end{array}$ & $\begin{array}{c}24.61 \\
* *\end{array}$ & $\begin{array}{c}33.99 \\
* *\end{array}$ & $\begin{array}{c}43.21 \\
* *\end{array}$ & $\begin{array}{c}41.24 \\
* *\end{array}$ & $\begin{array}{c}66.91 \\
* *\end{array}$ & $\begin{array}{c}182.2 \\
1 \\
* *\end{array}$ & $\begin{array}{c}121.90 \\
* *\end{array}$ & $\begin{array}{c}254.5 \\
7 \\
* *\end{array}$ & $\begin{array}{c}306.1 \\
8 \\
* *\end{array}$ & $\begin{array}{c}116.67 \\
* *\end{array}$ & $\begin{array}{c}\mathbf{5 . 6 5} \\
* *\end{array}$ & $\begin{array}{c}8.40 \\
* *\end{array}$ & 1.14 & $\begin{array}{c}3.96 \\
*\end{array}$ \\
\hline Error & 74 & 0.34 & 0.67 & 0.52 & 1.33 & 0.98 & 2.51 & 2.50 & 3.06 & $\begin{array}{c}37.94 \\
* *\end{array}$ & 2.06 & $\begin{array}{c}14.18 \\
* *\end{array}$ & $\begin{array}{c}16.88 \\
* *\end{array}$ & $\begin{array}{c}7.81 \\
* *\end{array}$ & 0.47 & 0.45 & 0.05 & 0.10 \\
\hline
\end{tabular}

*Significant at $5 \%$ level $* *$ Significant at $1 \%$ level

$\operatorname{DDd}_{1}=$ Degree of indetermination of pod maturity from $1^{\text {st }}$ flower $\left(D_{1}\right)$ to $90 \%$ pod maturity $\left(D_{3}\right)$

$\mathrm{DDd}_{2}=$ Degree of indetermination of pod maturity from $1^{\text {st }}$ pod maturity to $90 \%$ pods maturity

$\mathrm{DDh}_{1}=$ Degree of indetermination of plant height from $1^{\text {st }}$ flower to 1 st pods maturity

$\mathrm{DDh}_{2}=$ Degree of indetermination of plant height from $1^{\text {st }}$ flower to $90 \%$ pods maturity

$\mathrm{DDh}_{3}=$ Degree of indetermination of plant height from $1^{\text {st }}$ pod maturity to $90 \%$ pods maturity 
Table.2 Performance of greengram genotypes for different quantitative traits

\begin{tabular}{|c|c|c|c|c|c|c|c|c|c|c|c|c|c|c|c|c|c|c|}
\hline SI No & Genotypes & $\begin{array}{l}\text { Days to 1st } \\
\text { flowering } \\
\left(D_{1}\right)\end{array}$ & $\begin{array}{c}\text { days to } \\
50 \% \\
\text { flowering }\end{array}$ & $\begin{array}{l}\text { Days to } \\
\text { 1st pod } \\
\text { maturity } \\
\left(D_{2}\right)\end{array}$ & $\begin{array}{c}\text { DDd }_{1}= \\
\left(\text { (D3-D }_{1}\right) / \\
\text { D }_{3} * 100\end{array}$ & $\begin{array}{c}\text { Days to } \\
90 \% \text { pod } \\
\text { maturity } \\
\left(D_{3}\right)\end{array}$ & $\begin{array}{l}\mathbf{D D d}_{2}= \\
\left(\mathbf{D}_{3}-\mathbf{D}_{2}\right) / \\
\mathbf{D}_{3} * 100\end{array}$ & $\begin{array}{c}\text { Plant } \\
\text { height at } \\
1^{\text {st flower }} \\
\text { initiation } \\
\left(\mathbf{H}_{1}\right)\end{array}$ & $\begin{array}{c}\text { Plant } \\
\text { height at } \\
1^{\text {st pod }} \\
\text { maturity } \\
\left(\mathrm{H}_{2}\right)\end{array}$ & $\begin{array}{c}\mathrm{DDh}_{1}= \\
\left(\mathbf{H}_{2}-\mathbf{H}_{1}\right) / \\
\mathbf{H}_{2} * 100\end{array}$ & $\begin{array}{c}\text { Plant } \\
\text { height at } \\
90 \% \\
\text { pods } \\
\text { maturity } \\
\left(\mathrm{H}_{3}\right)\end{array}$ & $\begin{array}{c}\mathbf{D D h}_{2}= \\
\left(\mathbf{H}_{3}-\mathbf{H}_{1}\right) / \\
\mathbf{H}_{3} * 100\end{array}$ & $\begin{array}{c}\mathbf{D D h}_{3}= \\
\left(\mathbf{H}_{3}-\mathbf{H}_{2}\right) / \\
\mathbf{H}_{3} * 100\end{array}$ & $\begin{array}{c}\text { pods } \\
\text { plant }^{-1}\end{array}$ & $\begin{array}{c}\text { branches } \\
\text { plant }^{-1}\end{array}$ & $\begin{array}{l}\text { seeds } \\
\text { pod }^{-1}\end{array}$ & $\begin{array}{c}100 \text { seed } \\
\text { weight }\end{array}$ & $\begin{array}{r}\text { seed } \\
\text { yield } \\
\text { plant }\end{array}$ \\
\hline 1 & ML 818 & 44.67 & 54.33 & 58.00 & 36.19 & 70.00 & 17.14 & 20.13 & 28.33 & 28.93 & 48.44 & 58.43 & 41.52 & 4.67 & 3.33 & 11.33 & 3.27 & 5.79 \\
\hline 2 & KM 2241 & 45.00 & 52.33 & 57.33 & 33.82 & 68.00 & 15.69 & 18.16 & 23.72 & 23.44 & 35.61 & 49.00 & 33.37 & 13.00 & 6.67 & 10.00 & 4.19 & 7.35 \\
\hline 3 & MH 2-15 & 41.33 & 50.67 & 55.33 & 36.72 & 65.33 & 15.29 & 20.29 & 26.44 & 23.15 & 40.68 & 50.12 & 34.98 & 7.00 & 6.33 & 9.00 & 5.15 & 9.88 \\
\hline 4 & OUM 11-5 & 46.00 & 57.33 & 57.67 & 34.29 & 70.00 & 17.62 & 20.37 & 31.98 & 36.28 & 43.17 & 52.78 & 25.91 & 19.00 & 5.33 & 10.67 & 4.06 & 7.17 \\
\hline 5 & HUM 1 & 41.33 & 53.33 & 54.67 & 39.81 & 68.67 & 20.39 & 24.67 & 32.52 & 24.02 & 49.92 & 50.58 & 34.85 & 15.33 & 7.33 & 10.67 & 4.99 & 9.12 \\
\hline 6 & COGG 912 & 44.33 & 56.33 & 58.00 & 39.54 & 73.33 & 20.91 & 23.50 & 35.17 & 33.16 & 50.17 & 53.15 & 29.87 & 7.67 & 4.33 & 9.67 & 4.26 & 8.59 \\
\hline 7 & Pant M-5 & 47.00 & 55.67 & 59.33 & 37.61 & 75.33 & 21.23 & 13.50 & 20.40 & 33.83 & 35.67 & 62.16 & 42.80 & 9.00 & 3.67 & 8.00 & 4.33 & 8.02 \\
\hline 8 & Pusa 0672 & 48.33 & 59.67 & 62.33 & 33.49 & 72.67 & 14.22 & 17.50 & 22.83 & 23.30 & 38.33 & 54.32 & 40.44 & 4.33 & 4.33 & 8.00 & 3.81 & 6.31 \\
\hline 9 & Pant M-4 & 48.67 & 60.67 & 62.67 & 30.48 & 70.00 & 10.48 & 20.50 & 27.06 & 24.24 & 36.00 & 43.04 & 24.82 & 4.56 & 7.33 & 12.00 & 3.79 & 7.55 \\
\hline 10 & IPM 02-3 & 44.00 & 55.67 & 57.67 & 38.32 & 71.33 & 19.16 & 17.17 & 28.67 & 40.13 & 48.17 & 64.37 & 40.51 & 8.55 & 5.33 & 10.67 & 4.76 & 8.62 \\
\hline 11 & BM 4 & 42.33 & 54.33 & 55.67 & 39.91 & 70.67 & 20.98 & 23.67 & 36.33 & 35.00 & 60.89 & 61.14 & 40.34 & 16.00 & 6.67 & 9.67 & 3.64 & 7.39 \\
\hline 12 & AKM 8802 & 41.33 & 52.67 & 54.00 & 43.89 & 73.67 & 26.69 & 16.67 & 22.33 & 25.36 & 33.33 & 49.95 & 32.92 & 11.67 & 4.33 & 12.33 & 3.21 & 6.72 \\
\hline 13 & IPM 2-14 & 45.00 & 55.67 & 56.67 & 38.91 & 73.67 & 23.07 & 24.83 & 32.78 & 24.28 & 42.33 & 41.37 & 22.59 & 9.19 & 4.67 & 8.33 & 3.24 & 7.00 \\
\hline 14 & IPM 312-19 & 43.00 & 54.67 & 55.67 & 37.38 & 68.67 & 18.93 & 19.33 & 28.50 & 32.13 & 43.44 & 55.49 & 34.39 & 27.78 & 7.67 & 11.67 & 3.67 & 6.76 \\
\hline 15 & IPM 312-20 & 42.00 & 53.33 & 55.67 & 39.12 & 69.00 & 19.31 & 22.33 & 33.33 & 32.46 & 49.33 & 54.72 & 32.50 & 29.89 & 7.67 & 13.00 & 4.22 & 8.20 \\
\hline 16 & IPM 14-7 & 43.33 & 56.33 & 59.67 & 42.48 & 75.33 & 20.80 & 22.33 & 34.67 & 35.57 & 41.67 & 46.40 & 16.82 & 16.64 & 5.33 & 9.33 & 3.68 & 7.71 \\
\hline 17 & GGG 1 & 45.00 & 56.33 & 57.33 & 40.53 & 75.67 & 24.23 & 22.33 & 30.00 & 25.50 & 36.00 & 37.96 & 16.60 & 7.22 & 4.33 & 8.33 & 3.85 & 7.63 \\
\hline 18 & MH 1142 & 43.67 & 55.67 & 57.33 & 39.32 & 72.00 & 20.33 & 20.83 & 29.33 & 28.95 & 37.33 & 44.20 & 21.39 & 14.44 & 3.67 & 8.33 & 3.71 & 7.64 \\
\hline 19 & RMG 1087 & 41.00 & 52.67 & 54.33 & 40.30 & 68.67 & 20.87 & 21.33 & 29.67 & 27.95 & 32.33 & 33.98 & 8.28 & 14.89 & 5.67 & 10.67 & 4.00 & 7.88 \\
\hline 20 & RMG 1092 & 46.33 & 58.33 & 61.00 & 38.77 & 75.67 & 19.37 & 28.33 & 31.00 & 8.38 & 37.33 & 23.96 & 16.61 & 18.67 & 7.33 & 10.67 & 3.32 & 7.01 \\
\hline 21 & COGG 13-19 & 44.00 & 55.33 & 55.67 & 41.85 & 75.67 & 26.43 & 22.33 & 30.20 & 26.12 & 37.67 & 40.79 & 19.90 & 13.22 & 5.33 & 8.67 & 4.17 & 8.68 \\
\hline
\end{tabular}




\begin{tabular}{|c|c|c|c|c|c|c|c|c|c|c|c|c|c|c|c|c|c|c|}
\hline $\begin{array}{l}\text { SI } \\
\text { No }\end{array}$ & Genotypes & $\begin{array}{l}\text { Days to } \\
\text { 1st } \\
\text { flowering } \\
\left(D_{1}\right)\end{array}$ & $\begin{array}{c}\text { days to } \\
50 \% \\
\text { flowering }\end{array}$ & $\begin{array}{l}\text { Days to } \\
\text { 1st pod } \\
\text { maturi } \\
\operatorname{ty}\left(D_{2}\right)\end{array}$ & $\begin{array}{c}\mathrm{DDd}_{1}= \\
\left(\mathrm{D3}^{-}\right. \\
\left.\mathrm{D}_{1}\right) / \mathrm{D}_{3} * 1 \\
00\end{array}$ & $\begin{array}{c}\text { Days to } \\
90 \% \\
\text { pod } \\
\text { maturi } \\
\text { ty }\left(D_{3}\right)\end{array}$ & $\begin{array}{c}\mathbf{D D d}_{2}= \\
\left(\mathbf{D}_{3^{-}}\right. \\
\left.\mathbf{D}_{2}\right) / \\
\mathbf{D}_{3} * 100\end{array}$ & $\begin{array}{l}\text { Plant } \\
\text { height } \\
\text { at } 1^{\text {st }} \\
\text { flower } \\
\text { initiati } \\
\text { on }\left(\mathbf{H}_{1}\right)\end{array}$ & $\begin{array}{c}\text { Plant } \\
\text { height } \\
\text { at } 1^{\text {st }} \\
\text { pod } \\
\text { maturi } \\
\text { ty }\left(\mathrm{H}_{2}\right)\end{array}$ & $\begin{array}{c}\mathbf{D D h}_{1}= \\
\left(\mathbf{H}_{2^{-}}\right. \\
\left.\mathbf{H}_{1}\right) / \\
\mathbf{H}_{2} * 100\end{array}$ & $\begin{array}{c}\text { Plant } \\
\text { height at } \\
90 \% \text { pods } \\
\text { maturity } \\
\left(\mathrm{H}_{3}\right)\end{array}$ & $\begin{array}{c}\mathrm{DDh}_{2}= \\
\left(\mathrm{H}_{3^{-}}\right. \\
\left.\mathrm{H}_{1}\right) / \mathbf{H}_{3} \\
* \mathbf{1 0 0}\end{array}$ & $\begin{array}{c}\mathrm{DDh}_{3}= \\
\left(\mathrm{H}_{3^{-}}\right. \\
\left.\mathrm{H}_{2}\right) / \mathrm{H}_{3} \\
* 100\end{array}$ & $\begin{array}{l}\text { pods } \\
\text { plant }^{-1}\end{array}$ & $\begin{array}{c}\text { branches } \\
\text { plant }^{-1}\end{array}$ & $\begin{array}{l}\text { seeds } \\
\text { pod }^{-1}\end{array}$ & $\begin{array}{c}100 \\
\text { seed } \\
\text { weight }\end{array}$ & $\begin{array}{c}\text { seed } \\
\text { yield } \\
\text { plant }^{-1}\end{array}$ \\
\hline 22 & PM 14-3 & 42.00 & 53.67 & 56.33 & 40.28 & 70.33 & 19.91 & 17.33 & 28.00 & 37.99 & 38.67 & 55.36 & 27.53 & 10.55 & 6.33 & 10.33 & 4.25 & 7.84 \\
\hline 23 & PM 14-11 & 43.00 & 55.67 & 56.67 & 40.28 & 72.00 & 21.30 & 23.67 & 34.00 & 30.07 & 40.00 & 40.79 & 15.07 & 6.67 & 4.33 & 9.67 & 4.01 & 8.54 \\
\hline 24 & AKM 12-28 & 42.00 & 53.00 & 56.67 & 35.05 & 64.67 & 12.37 & 23.33 & 38.17 & 38.89 & 42.67 & 45.35 & 10.53 & 20.11 & 7.33 & 12.67 & 4.75 & 9.37 \\
\hline 25 & AKM 12-24 & 42.00 & 53.00 & 58.33 & 35.39 & 65.00 & 10.26 & 29.67 & 36.33 & 18.21 & 43.33 & 31.29 & 15.85 & 23.22 & 7.33 & 12.67 & 5.38 & 9.92 \\
\hline 26 & TMB 126 & 45.00 & 55.33 & 57.67 & 37.79 & 72.33 & 20.28 & 13.67 & 20.67 & 33.64 & 30.33 & 54.85 & 31.87 & 9.66 & 6.33 & 8.67 & 3.33 & 6.05 \\
\hline 27 & SKNM 1502 & 47.00 & 58.67 & 61.00 & 38.69 & 76.67 & 20.43 & 20.89 & 27.83 & 24.94 & 34.33 & 39.18 & 18.94 & 8.47 & 4.33 & 7.67 & 3.38 & 6.05 \\
\hline 28 & SKNM 1504 & 47.33 & 58.67 & 59.67 & 34.56 & 72.33 & 17.51 & 20.78 & 31.40 & 33.87 & 39.80 & 47.80 & 21.11 & 7.91 & 4.00 & 8.67 & 4.03 & 6.83 \\
\hline 29 & LGG 607 & 42.33 & 55.00 & 56.67 & 38.35 & 68.67 & 17.47 & 17.87 & 33.40 & 46.53 & 39.80 & 55.12 & 16.08 & 12.07 & 5.00 & 9.00 & 3.93 & 7.93 \\
\hline 30 & JAUM 0936 & 47.33 & 56.33 & 59.67 & 34.86 & 72.67 & 17.88 & 18.00 & 31.47 & 42.82 & 35.87 & 49.81 & 12.23 & 9.93 & 6.02 & 8.00 & 3.22 & 6.94 \\
\hline 31 & NMK 15-08 & 48.67 & 60.33 & 62.33 & 35.40 & 75.33 & 17.25 & 29.00 & 39.53 & 26.65 & 49.10 & 40.93 & 19.47 & 7.67 & 4.33 & 8.67 & 3.75 & 7.74 \\
\hline 32 & KM 2355 & 41.33 & 53.33 & 54.67 & 39.80 & 68.67 & 20.39 & 18.23 & 32.75 & 44.39 & 38.33 & 52.49 & 14.47 & 13.40 & 7.33 & 13.00 & 5.40 & 10.22 \\
\hline 33 & ML 2479 & 47.00 & 59.33 & 59.67 & 34.72 & 72.00 & 17.13 & 19.97 & 33.20 & 37.90 & 47.81 & 58.15 & 30.62 & 6.07 & 6.00 & 9.33 & 3.95 & 7.94 \\
\hline 34 & SML 1808 & 46.33 & 55.67 & 56.67 & 39.30 & 76.33 & 25.76 & 18.53 & 24.78 & 24.35 & 41.67 & 55.28 & 40.14 & 8.60 & 3.81 & 9.33 & 4.10 & 9.33 \\
\hline 35 & COGG 13-39 & 40.67 & 51.00 & 55.33 & 41.34 & 69.33 & 20.17 & 25.13 & 32.93 & 23.65 & 37.07 & 32.15 & 11.07 & 11.00 & 5.67 & 13.00 & 3.78 & 8.43 \\
\hline 36 & RMG 1097 & 47.67 & 59.67 & 62.67 & 34.40 & 72.67 & 13.76 & 22.80 & 30.20 & 24.43 & 36.40 & 37.36 & 17.04 & 6.00 & 3.67 & 8.00 & 3.46 & 7.00 \\
\hline 37 & SGC 16 & 48.00 & 58.33 & 61.67 & 37.39 & 76.67 & 19.57 & 16.87 & 24.47 & 30.96 & 31.27 & 46.10 & 21.82 & 7.53 & 4.11 & 8.00 & 2.93 & 6.03 \\
\hline \multirow[t]{6}{*}{38} & SGC 20 & 47.33 & 58.67 & 60.33 & 38.53 & 77.00 & 21.65 & 19.73 & 24.83 & 20.48 & 36.13 & 45.35 & 31.26 & 3.93 & 3.93 & 8.67 & 3.13 & 6.27 \\
\hline & Mean & 44.54 & 55.71 & 57.95 & 37.86 & 71.74 & 19.11 & 20.94 & 29.98 & 29.79 & 40.54 & 47.77 & 25.43 & 11.72 & 5.44 & 9.90 & 3.95 & 7.72 \\
\hline & C.V. & 1.31 & 1.47 & 1.24 & 3.05 & 1.38 & 8.29 & 7.55 & 5.83 & 20.68 & 3.54 & 7.88 & 16.16 & 23.83 & 12.61 & 6.74 & 5.55 & 4.02 \\
\hline & S.E. & 0.34 & 0.47 & 0.41 & 0.67 & 0.57 & 0.92 & 0.91 & 1.01 & 3.56 & 0.83 & 2.17 & 2.37 & 1.61 & 0.40 & 0.39 & 0.13 & 0.18 \\
\hline & C.D. 5\% & 0.95 & 1.33 & 1.17 & 1.88 & 1.61 & 2.58 & 2.57 & 2.84 & 10.02 & 2.34 & 6.13 & 6.68 & 4.55 & 1.12 & 1.09 & 0.36 & 0.51 \\
\hline & Range & $\begin{array}{l}40.67- \\
48.67\end{array}$ & $\begin{array}{l}50.67- \\
60.67\end{array}$ & $\begin{array}{c}54- \\
62.67\end{array}$ & $\begin{array}{l}30.48- \\
43.89\end{array}$ & $\begin{array}{c}64.67- \\
77\end{array}$ & $\begin{array}{l}10.26- \\
26.69\end{array}$ & $\begin{array}{l}13.5- \\
29.67\end{array}$ & $\begin{array}{l}20.4- \\
39.53\end{array}$ & $\begin{array}{l}8.38- \\
46.53\end{array}$ & $\begin{array}{l}30.33- \\
60.89\end{array}$ & $\begin{array}{l}23.96- \\
64.37\end{array}$ & $\begin{array}{l}8.28- \\
42.8\end{array}$ & $\begin{array}{l}3.93- \\
29.89\end{array}$ & $\begin{array}{l}3.33- \\
7.67\end{array}$ & $7.67-13$ & $\begin{array}{c}2.93- \\
5.4\end{array}$ & $\begin{array}{l}5.79- \\
10.22\end{array}$ \\
\hline
\end{tabular}


Table.3 Genetic parameters of variations for different traits in greengram

\begin{tabular}{|c|c|c|c|c|c|c|c|c|c|c|c|c|c|c|c|c|c|}
\hline $\begin{array}{l}\text { Genetic } \\
\text { parameters }\end{array}$ & $\begin{array}{l}\text { Days to } \\
\text { 1st } \\
\text { flowering } \\
\left(D_{1}\right)\end{array}$ & $\begin{array}{l}\text { Days to } \\
50 \% \\
\text { flowering }\end{array}$ & $\begin{array}{l}\text { Days to } \\
\text { 1st pod } \\
\text { maturity } \\
\left(D_{2}\right)\end{array}$ & $\operatorname{DDd}_{1}$ & $\begin{array}{l}\text { Days to } \\
90 \% \\
\text { pod } \\
\text { maturity } \\
\left(\mathrm{D}_{3}\right)\end{array}$ & $\mathrm{DDd}_{2}$ & $\begin{array}{l}\text { Plant } \\
\text { height at } \\
1^{\text {st flower }} \\
\text { initiation } \\
\left(\mathrm{H}_{1}\right)\end{array}$ & $\begin{array}{l}\text { Plant } \\
\text { height at } \\
1^{\text {st }} \text { pod } \\
\text { maturity } \\
\left(\mathrm{H}_{2}\right)\end{array}$ & $\mathrm{DDh}_{1}$ & $\begin{array}{l}\text { Plant } \\
\text { height at } \\
90 \% \\
\text { pods } \\
\text { maturity } \\
\left(\mathrm{H}_{3}\right)\end{array}$ & $\mathrm{DDh}_{2}$ & $\mathrm{DDh}_{3}$ & $\begin{array}{l}\text { Number } \\
\text { of pods } \\
\text { per } \\
\text { plant }\end{array}$ & $\begin{array}{l}\text { Number } \\
\text { of } \\
\text { branches } \\
\text { per } \\
\text { plant }\end{array}$ & $\begin{array}{l}\text { Seeds } \\
\text { per } \\
\text { pods }\end{array}$ & $\begin{array}{l}100 \\
\text { seed } \\
\text { weight } \\
(\mathrm{g})\end{array}$ & $\begin{array}{l}\text { seed } \\
\text { yield } \\
\text { per } \\
\text { plant } \\
(\mathrm{g})\end{array}$ \\
\hline GCV & 5.58 & 4.62 & 4.26 & 7.36 & 4.62 & 19.27 & 17.16 & 15.39 & 23.28 & 15.59 & 18.74 & 38.61 & 51.38 & 24.17 & 16.44 & 15.28 & 14.70 \\
\hline PCV & 5.74 & 4.85 & 4.44 & 7.96 & 4.83 & 20.98 & 18.75 & 16.45 & 31.14 & 15.99 & 20.33 & 41.85 & 56.64 & 27.26 & 17.77 & 16.26 & 15.24 \\
\hline $\mathbf{H}^{2}$ (bs) & 0.95 & 0.91 & 0.92 & 0.85 & 0.92 & 0.84 & 0.84 & 0.87 & 0.56 & 0.95 & 0.85 & 0.85 & 0.82 & 0.79 & 0.85 & 0.88 & 0.93 \\
\hline GAM & 11.19 & 9.07 & 8.43 & 14.00 & 9.13 & 36.47 & 32.37 & 29.64 & 35.86 & 31.32 & 35.58 & 73.37 & 96.02 & 44.14 & 31.33 & 29.58 & 29.20 \\
\hline
\end{tabular}

Table.4 Cluster composition of Greengram Genotypes (Tocher's Method)

\begin{tabular}{|c|c|c|c|}
\hline SI. No & Cluster & Number of genotypes & Name of genotypes \\
\hline 1 & Cluster I & 23 & $\begin{array}{l}\text { SGC20, SKNM1502, SGC16, RMG1097, Pusa0672 SKNM1504, JAUM0936, TMB126, GGG1, } \\
\text { MH1142, IPM2-14, OUM11-5, IPM14-7, ML2479, RMG1092, PM14-3, PM14-11, LGG607, } \\
\text { COGG13-19, COGG912, IPM312-19, ML818, SML1808 }\end{array}$ \\
\hline 2 & Cluster II & 6 & COGG13-39, RMG1087, KM2355 , HUM1, PM312-20, IPM02-3 \\
\hline 3 & Cluster III & 3 & AKM12-24, АKM12-28, MH2-15 \\
\hline 4 & Cluster IV & 2 & AKM8802, KM2241 \\
\hline 5 & Cluster V & 1 & BM4 \\
\hline 6 & Cluster VI & 1 & NMK15-08 \\
\hline 7 & Cluster VII & 1 & PantM-4 \\
\hline 8 & Cluster VIII & 1 & PantM-5 \\
\hline
\end{tabular}


Table.5 Intra- Cluster (Diagonal) And Inter- Cluster Distances for eight clusters in Greengram

\begin{tabular}{|c|c|c|c|c|c|c|c|c|}
\hline & Cluster I & Cluster II & Cluster III & Cluster IV & Cluster V & Cluster VI & Cluster VII & Cluster VIII \\
\hline Cluster I & 201.58 & 322.92 & 761.70 & 265.40 & 608.98 & 277.13 & 255.01 & 278.43 \\
\hline Cluster II & & 194.20 & 445.03 & 296.68 & 417.32 & 523.03 & 430.63 & 502.78 \\
\hline Cluster III & & & 186.73 & 561.34 & 840.60 & 1046.15 & 719.35 & 910.01 \\
\hline Cluster IV & & & & 205.62 & 746.07 & 574.16 & 322.14 & 307.15 \\
\hline Cluster V & & & & & 0.00 & 688.80 & 799.51 & 984.36 \\
\hline Cluster VI & & & & & & 0.00 & 294.88 & 346.23 \\
\hline Cluster VII & & & & & & & 0.00 & 346.11 \\
\hline Cluster VIII & & & & & & & & 0.00 \\
\hline
\end{tabular}

Table.6 Mean performance of the clusters with respect to different traits

\begin{tabular}{|c|c|c|c|c|c|c|c|c|c|c|c|c|c|c|c|c|c|}
\hline Cluster & $\begin{array}{l}\text { Days } \\
\text { to 1st } \\
\text { flower } \\
\text { ing } \\
\left(D_{1}\right)\end{array}$ & $\begin{array}{l}\text { Days to } \\
50 \% \\
\text { flowering }\end{array}$ & $\begin{array}{l}\text { Days to } \\
\text { 1st pod } \\
\text { maturity } \\
\left(\mathrm{D}_{2}\right)\end{array}$ & DDd $_{1}$ & $\begin{array}{l}\text { Days to } \\
90 \% \text { pod } \\
\text { maturity } \\
\left(D_{3}\right)\end{array}$ & $\mathrm{DDd}_{2}$ & $\begin{array}{l}\text { Plant } \\
\text { height at } \\
1^{\text {st }} \text { flower } \\
\text { initiation } \\
\left(\mathrm{H}_{1}\right)\end{array}$ & $\begin{array}{l}\text { Plant } \\
\text { height at } \\
1^{\text {st }} \text { pod } \\
\text { maturity } \\
\left(\mathrm{H}_{2}\right)\end{array}$ & $\mathbf{D D h}_{1}$ & $\begin{array}{l}\text { Plant } \\
\text { height at } \\
90 \% \text { pods } \\
\text { maturity } \\
\left(\mathrm{H}_{3}\right)\end{array}$ & $\mathbf{D D h}_{2}$ & $\mathbf{D D h}_{3}$ & $\begin{array}{l}\text { Numbe } \\
\text { r of } \\
\text { pods } \\
\text { per } \\
\text { plant }\end{array}$ & $\begin{array}{l}\text { Numbe } \\
\mathbf{r} \text { of } \\
\text { branche } \\
\text { s per } \\
\text { plant }\end{array}$ & $\begin{array}{l}\text { Seeds } \\
\text { per } \\
\text { pods }\end{array}$ & $\begin{array}{l}100 \\
\text { seed } \\
\text { weigh } \\
t(g)\end{array}$ & $\begin{array}{l}\text { seed } \\
\text { yield } \\
\text { per } \\
\text { plant } \\
\text { (g) } \\
\end{array}$ \\
\hline Cluster I & 45.39 & 56.74 & 58.61 & 37.91 & 73.15 & 19.81 & 20.52 & 29.52 & 30.03 & 39.48 & 47.57 & 24.77 & 10.45 & 4.94 & 9.15 & 3.69 & 7.26 \\
\hline ClusterII & 41.72 & 53.22 & 55.39 & 39.78 & 69.28 & 20.05 & 21.48 & 31.65 & 32.10 & 42.53 & 48.05 & 23.61 & 13.51 & 6.50 & 11.83 & 4.53 & 8.74 \\
\hline ClusterIII & 41.78 & 52.22 & 56.78 & 35.72 & 65.00 & 12.64 & 24.43 & 33.65 & 26.75 & 42.23 & 42.25 & 20.46 & 16.78 & 7.00 & 11.44 & 5.10 & 9.72 \\
\hline Cluster IV & 43.17 & 52.50 & 55.67 & 38.86 & 70.83 & 21.19 & 17.42 & 23.03 & 24.40 & 34.47 & 49.47 & 33.15 & 12.33 & 5.50 & 11.17 & 3.70 & 7.04 \\
\hline Cluster V & 42.33 & 54.33 & 55.67 & 39.91 & 70.67 & 20.98 & 23.67 & 36.33 & 35.00 & 60.89 & 61.14 & 40.34 & 16.00 & 6.67 & 9.67 & 3.64 & 7.39 \\
\hline Cluster VI & 48.67 & 60.33 & 62.33 & 35.40 & 75.33 & 17.25 & 29.00 & 39.53 & 26.65 & 49.10 & 40.93 & 19.47 & 7.67 & 4.33 & 8.67 & 3.75 & 7.74 \\
\hline Cluster VII & 48.67 & 60.67 & 62.67 & 30.48 & 70.00 & 10.48 & 20.50 & 27.06 & 24.24 & 36.00 & 43.04 & 24.82 & 4.56 & 7.33 & 12.00 & 3.79 & 7.55 \\
\hline Cluster VIII & 47.00 & 55.67 & 59.33 & 37.61 & 75.33 & 21.23 & 13.50 & 20.40 & 33.83 & 35.67 & 62.16 & 42.80 & 9.00 & 3.67 & 8.00 & 4.33 & 8.02 \\
\hline
\end{tabular}


Table.7 Contribution of different quantitative traits to diversity in greengram following Singh (1981)

\begin{tabular}{|c|c|c|c|c|c|c|c|c|c|c|c|c|c|c|c|c|c|}
\hline & 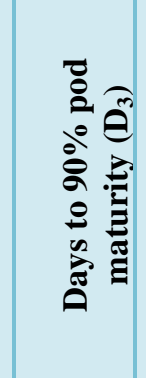 & 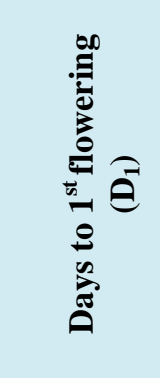 & 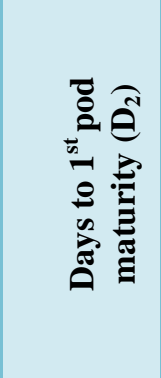 & 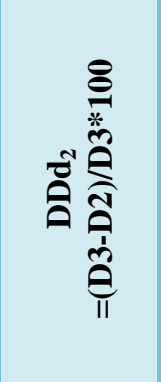 & 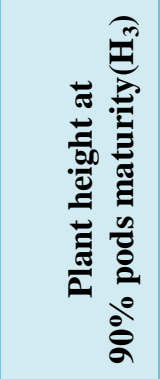 & ت气 & 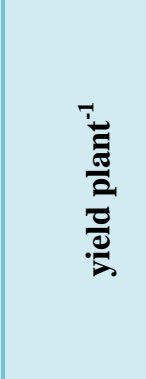 & 家 & 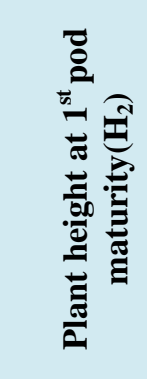 & 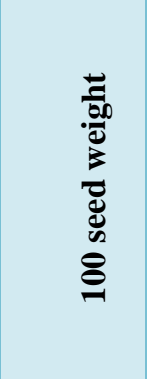 & 完 & 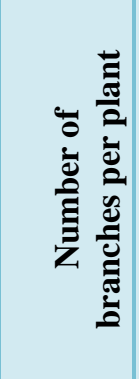 & 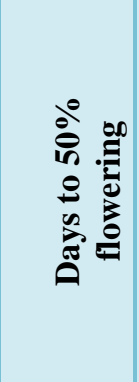 & 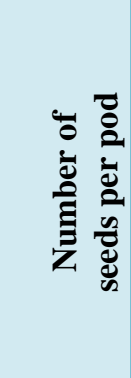 & 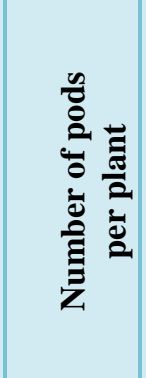 & 宣 & 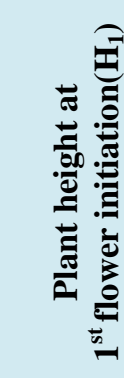 \\
\hline Singh statistic & $\begin{array}{c}406455.08 \\
3\end{array}$ & 228067.716 & 202363.781 & 133934.342 & 70557.460 & 42774.243 & 24332.463 & 21713.688 & 14338.796 & 12727.118 & 9220.979 & 7650.928 & 4324.299 & 3930.149 & 3537.430 & 234.366 & 159.715 \\
\hline Proportion & 0.343 & 0.192 & 0.171 & 0.113 & 0.059 & 0.036 & 0.021 & 0.018 & 0.012 & 0.011 & 0.008 & 0.006 & 0.004 & 0.003 & 0.003 & 0.000 & 0.000 \\
\hline $\begin{array}{l}\text { Cumulative } \\
\text { proportion }\end{array}$ & 0.343 & 0.535 & 0.705 & 0.818 & 0.878 & 0.914 & 0.934 & 0.953 & 0.965 & 0.976 & 0.983 & 0.990 & 0.993 & 0.997 & 1.000 & 1.000 & 1.000 \\
\hline
\end{tabular}


The better genotypes might be selected for most of characters on the basis of mean performance in the cluster.

Following Singh's (1981) approach used to identify the character contributing the most to the overall divergence, highest contribution was by days to $90 \%$ pod maturity followed by days to first flowering and days to first pod maturity (Table 7). Contributions were negligible by degree of indetermination of plant height from first flower to $90 \%$ pod maturity and plant height at first flower initiation. No previous reports were seen in relation to present findings for days to maturity towards the contribution of divergence, indicating careful consideration of the present results.

It is concluded from the present study, the best genotypes for yield and yield attributing characters identified were KM 2355, AKM 12-24, AKM 12-28, MH 2-15, IPM 312-20 and HUM 1. Pant M-4 was the most determinate at $90 \%$ pod maturity from first flower. RMG 1092 was the most determinate for plant height from first flower to first pod and $90 \%$ pod maturity. RMG 1087 was determinate for plant height from first pod maturity to $90 \%$ pod maturity. Based on the clustering pattern, cluster distance and cluster mean genotype PantM-4, AKM 12-24, AKM 12-28, MH 2-15, KM 2355, IPM 312-20 and COGG 13-39 could be useful for exploitation of hybrid vigor and for getting good recombinant. Days to $90 \%$ pod maturity, days to first flowering and days to first pod maturity showed high contribution toward genetic divergence.Sufficient variation for yield and synchronous maturity was observed which justifies scope for future breeding.

\section{Acknowledgement}

The authors are grateful to RARS, Shillongoni for providing the experimental material and also to the Department of Plant Breeding and Genetics, Assam Agricultural University, Jorhat for letting them carry out the research work efficiently.

\section{References}

Anand, G., Anandhi, K. and Paulpandi, V.K. (2015). Genetic variability, correlation and path analysis for yield and yield components in F6 families of Greengram (Vignaradiata (L.) Wilczek) under rainfed condition. Electronic Journal of Plant Breeding, 7: 434-437.

Anonymous (2018).https://farmer.gov.in/FinalReter ospectsProspectsofPulsesretrievedon 1 st Nov, 2018.

Anonymous (2019). http://dpd.gov.in/ Varieties/Mungbean\%20varieties.pdfret rieved on $1^{\text {st }}$ June, 2019.

Baisakh, B., Swain, S.C., Panigrahi, K.K., Das, T.R. and Mohanty, A. (2016). Estimation of Genetic Variability and Character Association in Micro Mutant Lines of Greengram [Vigna radiata (L.) Wilczek] for Yield Attributes and Cold Tolerance. Legume Genomics and Genetics, 77, 1-9.

Begum, S., Islam, M.A; Prodhan, A.K.M.A. (2007) Anatomy of rachis of the inflorescence in pigeon pea.International Journal of Botany,3: 85-90

Bisht, I. S., Bhat, K. V., Lakhanpaul, S., Latha, M., Jayan, P. K., Biswas, B. K. and Singh, A. K. (2005). Diversity and genetic resources of wild Vigna species in India. Genetic Resources and Crop Evolution. 52:53-68.

Bisht, I. S., Mahajan, R. K. and Patel, D. P. (1998). The use of characterisation data to establish the Indian mungbean core collection and assessment of genetic diversity. Genetic Resources and Crop Evolution. 45(2), 127-133 
Burton, G.W. and Devane, E.H. (1953). Estimating heritability in tall fescue (Festuca arundinacea) from replicated clonal material. Agronomy Journal.45: 478-481.

Bushby, H. and Lawn, R. (1992). Accumulation and partitioning of nitrogen and dry matter by contrasting genotypes of mungbean (Vignaradiata L. Wilczek). Australian Journal Agricultural Research. 43: 1609-1628

da Silva, F.A., Corrêa, A.M., Teodoro, P.E., Lopes, K.V., and Corrêa, C.C.G., (2017) Genetic divergence in the common bean (Phaseolus vulgaris L.) in the Cerrado-Pantanalecotone. Genet. Mol. Res., 16(1)

Dikshit, H.K., Singh, B.B. and Dua, R.R. (2002). Genetic variation in mungbean. Indian Journal of Pulses Research. 15:125-127.

Egli, D. and Bruening, W. (2006). Fruit development and reproductive survival in soybean: Position and age effects. Field Crops Research, 98: 195-202.

Fakir, M.S.A. (1997) A study on morphophysiological selection criteria related to yield in pigeonpea. Ph.D Dissertation, Department of Plant Science, University of West Indies, St. Augustine, Trinidad, p 72

Garg, G.K., Verma, P.K and Kesh, H. (2017).Multivariate analysis in mungbean (Vigna radiata) for genetic diversity under rainfed condition. Forage Research. 43 : 156-159.

Ginwal H.S., Rawat P.S., Srivastava R.L. (2004) Seed source variation in growth performance and oil yield of Jatropha curcas Linn.in Central India. Silvaegenetica.53: 186-192.

Hemavathy T. A., Shunmugavalli N. and Anand G. (2015). Genetic variability, correlation and path co-efficient studies on yield and its components in mungbean [Vigna radiata (L.)
Wilczek]. Legume Research.38:442446.

Johnson, H.W., Robinson, H.F. and Comstock, R.E. (1955).Estimates of genetic and environmental variability in soybeans. Agronomy Journal.47(7): 314-318.

Idress, A., Sadiq, M.S., Hanif, M., Abbas, G. and Haider, S. (2006). Genetic Analysis and Path Coefficient Analysis in Mutated Generation of Mungbean [Vigna radiata (L.) Wilczek]. Agricultural Research. 44: 181-191.

Khattak, G. S. S., Haq, M. A., Ashraf, M. and Tahir, G. R. (2001). Genetic Basis of Synchrony in Pod Maturity in Mungbean (Vigna radiata (L.) Wilczek). Kasetsart Journal (Natural Science), 35: 1-7.

Khattak, G.S.S., Ashraf, M.W. and Zamir, R. (2004). Gene Action for Synchrony In Pod Maturity And Indeterminate Growth Habit In Mungbean (Vigna radiata (L.) Wilczek). Pakistan Journal of Botany. 36: 589-594.

Kuroda, T., Saitoh, K., Mahmood, T, and Yanagawa, K. (1998) Differences in flowering habit between determinate and indeterminate types of soybean. Plant Production Sciences.1:18-24

Mehandi, S., Singh, C. M. and Kushwaha, V. K. (2013). Estimates of genetic variability and heritability for yield and yield component traits in Greengram [Vigna radiata (L.) Wilczek]. The Bioscan.8: 1481-1484.

Mishra, A. K., Singh, P. K., Kumar, R., Kumar, P., Singh, R., Kumar, A., \& Kumar, A. (2018).Genetic Divergence Study in Advance Indica Rice (Oryza sativa L.) Lines for Yield and Quality Attributes. International Journal of Current Microbiology and Applied Sciences. 7: 2924-2933.

Muthuswamy, A., Jamunarani, M. and Ramakrishnan, P. (2019). Genetic 
Variability, character Association and Path Analysis Studies in Green Gram (Vigna radiata (L.) Wilczek). International Journal of Current Microbiology and Applied Sciences. 8: 1136-1146.

Pavan, K., Reddy, P. and Mehta, C.M. (2019). Estimation of variability through genetic parameters and identification of superior pure lines for yield attributing traits in greengram [Vigna radiata (L.). Journal of Pharmacognosy and Phytochemistry. 8: 55-61.

Payasi, D.K. (2015). Genetic Variability Analysis for Seed Yield and its Components in Mungbean (Vigna radiata L. Wilczek). International Journal of Plant Breeding and Genetics. 9: $177-188$.

Rao, C.R. (1952). Advanced Statistical Methods in Biometrical Research. John Wiley and Sons, New York, pp.357369.

Raturi, A., Singh, S. K., Sharma, V. and Pathak, R. (2015). Genetic variability, heritability, genetic advance and path analysis in mungbean [Vigna radiata (L.)Wilczek]. Legume Research. 38: 157-163.

Reddy V.L.N., Reddisekhar, M., Reddy, K. R. and Reddy, K.H. (2003).Genetic variability for yield and its components in mungbean (Vigna radiata (L.) Wilczek). LegumeResearch.26:300-302.

Reddy, D.K.R., Venkateswarlu, O., Obaiah, M.C. and Jyothi, G.L.S. (2011).Studies on genetic variability, character association and path-coefficient analysis in greengram. Legume Research. 34:
202-206

Robinson, H.F., Comstock, R.E. and Harvey, P.H. (1949). Estimates of heritability and the degree of dominance in corn. Agronomy Journal.

Sharma-Natu, P. and Ghildiyal, M.C. (2005). Potential targets for improving photosynthesis and crop yield. Current Science. 88: 1918-1928

Shimelis, H. and Rhandzu, S. (2010). Variance components and heritabilities of yield and agronomic traits among cowpea genotypes. Euphytica, 176: 383-389.

Singh, C., Mishra, B.S. and Pandey, A. (2014).Pattern of agro-morphological trait relationship and genetic divergence in greengram [Vigna radiata (L.) Wilczek]. Electronic Journal of Plant Breeding. 5: 97-106.

Singh, D. (1981). The relative importance of characters affecting genetic divergence. Indian Journal of Genetics and Plant Breeding (The), 41(2), 237-245.

Singh, R., Ali, H. and Pathak, B. (2013). Nonhierarchical Euclidean cluster analysis in mungbean. Trends in Biosciences.3: 135-136.

Venkateswarlu, O. (2001). Genetic variability in green gram [Vigna radiata (L.) Wilczek]. Legume Research. 24: 69-70

Yeates, S., Lawn, R. and Adkins, S. (2000). Prediction of weather damage of mungbean seed in tropical Australia. I Relation between seed quality, weather and reproductive development. Australian Journal Agricultural Research, 51: 637-648.

\section{How to cite this article:}

Nivedita Talukdar, Hironyo Kr. Borah and Ramendra Nath Sarma. 2020. Genetic Variability of Traits Related to Synchronous Maturity in Greengram [Vigna radiata (L.) Wilczek]. Int.J.Curr.Microbiol.App.Sci. 9(01): 1120-1133. doi: https://doi.org/10.20546/ijcmas.2020.901.126 